\title{
Household Clusters of Severe Acute Respiratory Syndrome Coronavirus 2 (SARS-CoV-2) Infection in Suzhou, China
}

\author{
Lin Yao $\mathbb{D}^{1},{ }^{1}$ Peijun Tang $\mathbb{D}^{1},{ }^{1}$ Hui Jiang $\mathbb{D}^{2},{ }^{2}$ Binbin Gu $\mathbb{D}^{3},{ }^{3}$ Ping Xu $\mathbb{D}^{4},{ }^{4}$ Xiafang Wang $\mathbb{D}^{1}$ \\ Xin Yu $\mathbb{D}^{1},{ }^{1}$ Jianping Zhang $\mathbb{D}^{1},{ }^{1}$ Yu Pang $\mathbb{D}^{2},{ }^{2}$ and Meiying $W u\left(\mathbb{D}{ }^{1}\right.$ \\ ${ }^{1}$ Department of Pulmonary, The Fifth People's Hospital of Suzhou, The Affiliated Infectious Diseases Hospital of \\ Soochow University, Suzhou 215000, China \\ ${ }^{2}$ Department of Bacteriology and Immunology, Beijing Key Laboratory on Drug-Resistant Tuberculosis Research, \\ Beijing Chest Hospital, Capital Medical University/Beijing Tuberculosis \& Thoracic Tumor Research Institute, \\ Beijing 101149, China \\ ${ }^{3}$ Intensive Care Unit, The Fifth People's Hospital of Suzhou, The Affiliated Infectious Diseases Hospital of Soochow University, \\ Suzhou 215000, China \\ ${ }^{4}$ Department of Clinical Laboratory, The Fifth People's Hospital of Suzhou, The Affiliated Infectious Diseases Hospital of \\ Soochow University, Suzhou 215000, China
}

Correspondence should be addressed to Yu Pang; pangyupound@163.com and Meiying Wu; wu_my@126.com

Received 17 February 2021; Revised 27 May 2021; Accepted 12 September 2021; Published 16 October 2021

Academic Editor: San-Ji Gao

Copyright (C) 2021 Lin Yao et al. This is an open access article distributed under the Creative Commons Attribution License, which permits unrestricted use, distribution, and reproduction in any medium, provided the original work is properly cited.

Objectives. The severe acute respiratory syndrome coronavirus 2 (SARS-CoV-2) is an emerging virus causing substantial morbidity and mortality worldwide. We performed a cross-sectional investigation of SARS-CoV-2 clusters in Suzhou to determine the transmissibility of the virus among close contacts and to assess the demographic and clinical characteristics between index and secondary cases. Methods. We review the clustered patients with SARS-CoV-2 infections in Suzhou between 22 January and 29 February 2020. The demographic and clinical characteristics were compared between index and secondary cases. We calculated the basic reproduction number $\left(R_{0}\right)$ among close contacts with SLI model. Results. By 22 February, 87 patients with SARS-CoV-2 infection were reported, including 50 sporadic and 37 clustered cases, who were generated from 13 clusters. On admission, 5 (20.8\%) out of 24 secondary cases were asymptomatic. The male ratio of index cases was significantly higher than that of secondary cases. Additionally, the index cases were more likely to have fever and increased CRP levels than the secondary cases. The $R_{0}$ values of clusters displayed a significantly declining trend over time for all clusters. The relative risk of infection in blood-related contacts of cases versus unrelated contacts was 1.60 for SARS-CoV-2 (95\% CI: 0.42-2.95). Conclusions. In conclusion, SARS-CoV-2 has great person-to-person transmission capability among close contacts. The secondary cases are more prone to have mild symptoms than index cases. There is no increased RR of secondary infection in blood relatives versus unrelated contacts. The high rate of asymptomatic SARS-CoV-2 infections highlights the urgent need to enhance active case finding strategy for early detection of infectious patients.

\section{Introduction}

Since December 2019, China has been experiencing the emergence of a novel coronavirus, named as SARS-CoV-2, which can cause human coronavirus disease-19 (COVID$19)$, mainly presenting with respiratory disease, severe pneumonia, and multiple organ damage [1]. Although the origin of the infections has yet to be identified to date, the SARS-
CoV-2 is capable of human-to-human transmission [2]. Although there is occasional amplification in the healthcare settings, the predominant transmission potential of SARSCoV-2 occurs in the persons who have unprotected exposure to confirmed or subclinical cases [3]. Therefore, epidemiological analyses of detailed line lists of patients are of great importance to identify key parameters for understanding its infectivity, which is essential to formulate effective 


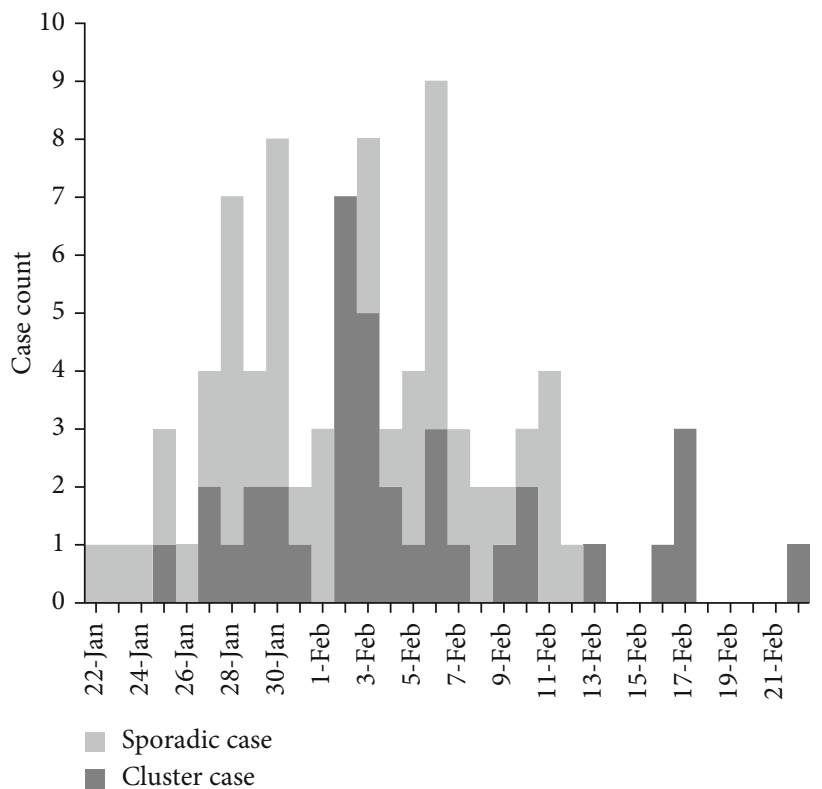

(a)

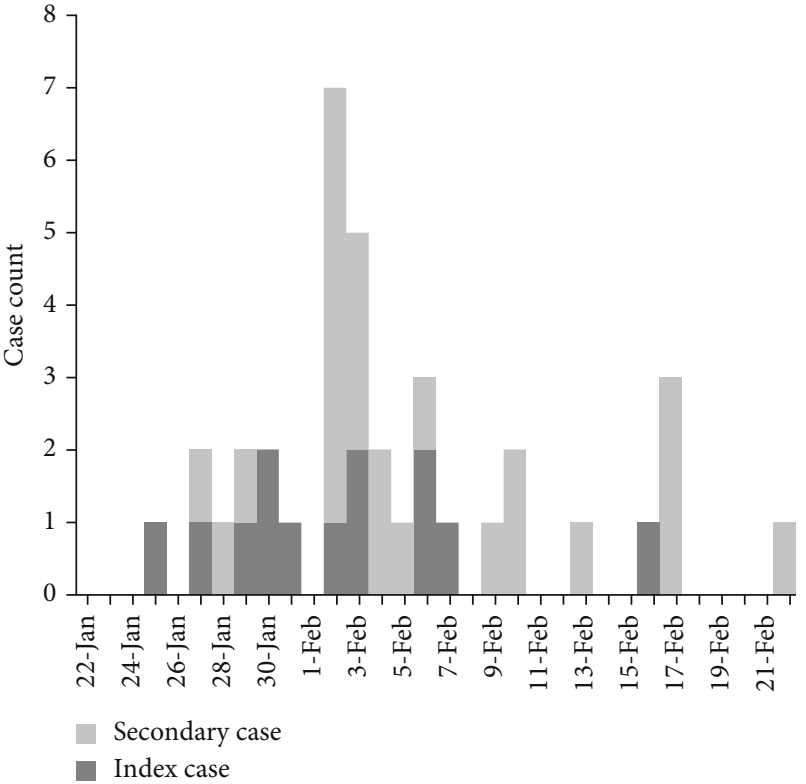

(b)

Figure 1: Cases of laboratory-confirmed SARS-CoV-2 infection reported between 22 January and 29 February 2020.

measures to protect close contacts against secondary infection.

The transmissibility of SARS-CoV-2 is the most important determinant of pandemic crisis and has become worrisome given the community outbreaks consisting of clusters with 2 or more epidemiologically linked cases [4]. Previous studies that have modeled the reproductive rate of SARSCoV-2 in human have been based on the notified and clinically apparent cases from epidemiological investigations or official websites on COVID-19 $[1,5,6]$. However, the exclusion of individuals with no or only mild respiratory symptoms becomes the barrier to conduct in-depth investigation of rate of secondary transmission in clusters. In addition, the meticulous analysis of clusters provides insights into not only the transmissibility of SARS-CoV-2 but also the familial susceptibility $[4,7]$. To address this concern, we performed a cross-sectional investigation of 13 SARS-CoV-2 clusters in Suzhou to determine the transmissibility of the virus among close contacts. The second objective of this study was to assess the demographic and clinical characteristics between index and secondary cases.

\section{Methods}

2.1. Patients. This study was conducted in the Fifth People's Hospital of Suzhou, which is a government-designated hospital for emerging infectious diseases. There have been 87 cases with laboratory-confirmed SARS-CoV-2 infections between 22 January and 29 February 2020, including 50 sporadic and 37 clustered cases. Laboratory-confirmed testing for SARS-CoV-2 infection was conducted using quantitative RT-PCR (qRT-PCR) by detection of SARS-CoV-2-specific fragment. The 37 patients associated with the 13 index patients were further included for epidemiological analysis. The routine blood counts and biochemical tests were assessed at the same day postsymptom onset by the Clinical Laboratory of the hospital. Demographic, clinical, and laboratory characteristics were obtained with data collection forms from electronic medical records. This study was approved by the Ethics Committee of the Fifth People's Hospital of Suzhou (No. 2020SZWY005). Written consent was obtained from each participant.

2.2. Definitions. All cases were diagnosed according to the detection SARS-CoV-2 in throat, nasopharyngeal, or cloacal swabs regardless of disease severity. The contacts were defined as individuals living in the same house or working in the same office. The blood-relative relationship was defined as parentoffspring, siblings, grandparent-grandchild, and uncle/auntniece/nephew, whereas the unrelated contact was defined as spouse, son/daughter-in-law, parent-in-law, workmate, and the other unrelated member [4]. We defined the index patient as the person with the earliest symptom onset date or laboratory-confirmed COVID-19 plus exposure in Wuhan.

2.3. Statistical Analysis. Chi-square or Fisher exact test was used, as appropriate, to compare continuous variables, which were presented with frequency (percentage). Continuous variables were summarized as means and standard deviations (SD), and the student $t$-test was used for comparison. $P$ values of $<0.05$ were considered statistically significantly. All calculations were carried out using SPSS 20.0 (SPSS Inc., Chicago, IL, USA).

In order to investigate whether there is genetic susceptibility to COVID-19, the data from the clustered cases were used to calculate the relative risk (RR) of infection of blood relatives versus unrelated contacts of the index case based on the assumption that the blood relatives had the same probability of detection infection as unrelated contacts. We followed the method used by Lemaitre and colleague [4]. 
TABle 1: Demographic and clinical characteristics of index and secondary cases in clusters.

\begin{tabular}{|c|c|c|c|c|}
\hline Characteristic & Total $(N=37, \%)$ & Cluster index cases $(n=13, \%)$ & Cluster secondary cases $(n=24, \%)$ & $P$ value \\
\hline Age (median, IQR) & & $49(39.5-61.5)$ & $36(22.5-46.8)$ & 0.017 \\
\hline Age group & & & & 0.054 \\
\hline $0-14$ & $5(13.5)$ & $0(0.0)$ & $5(20.8)$ & \\
\hline $15-24$ & $2(5.4)$ & $0(0.0)$ & $2(8.3)$ & \\
\hline $25-59$ & $23(62.2)$ & $9(69.2)$ & $14(58.3)$ & \\
\hline$\geq 60$ & $7(18.9)$ & $4(30.8)$ & $3(12.5)$ & \\
\hline Gender & & & & 0.040 \\
\hline Female & $17(45.9)$ & $3(23.1)$ & $14(58.3)$ & \\
\hline Male & $20(54.1)$ & $10(76.9)$ & $10(41.7)$ & \\
\hline Outcome & & & & 0.260 \\
\hline Survive & $21(56.8)$ & $9(69.2)$ & $12(50.0)$ & \\
\hline In treatment & $16(43.2)$ & $4(30.8)$ & $12(50.0)$ & \\
\hline \multicolumn{5}{|c|}{ Underlying chronic medical conditions } \\
\hline No & $32(86.5)$ & $11(84.6)$ & $21(87.5)$ & 0.808 \\
\hline Cardiovascular disease & $1(2.7)$ & $0(0.0)$ & $1(4.2)$ & 1.000 \\
\hline Diabetes mellitus & $1(2.7)$ & $1(7.7)$ & $0(0.0)$ & 0.351 \\
\hline Hypertension & $1(2.7)$ & $1(7.7)$ & $0(0.0)$ & 0.351 \\
\hline Others & $2(5.4)$ & $0(0.0)$ & $2(8.3)$ & 0.532 \\
\hline \multicolumn{5}{|l|}{ Onset symptom } \\
\hline Fever & $23(62.2)$ & $12(92.3)$ & $11(45.8)$ & 0.015 \\
\hline Cough & $23(62.2)$ & $11(84.6)$ & $12(50.0)$ & 0.086 \\
\hline Sore throat & $3(8.1)$ & $1(7.7)$ & $2(8.3)$ & 1.000 \\
\hline Runny nose & $3(8.1)$ & $0(0.0)$ & $3(12.5)$ & 0.485 \\
\hline Sputum production & $17(45.9)$ & $8(61.5)$ & $9(37.5)$ & 0.161 \\
\hline Vomiting & $1(2.7)$ & $1(7.7)$ & $0(0.0)$ & 0.351 \\
\hline Nausea & $1(2.7)$ & $1(7.7)$ & $0(0.0)$ & 0.351 \\
\hline Fatigue & $6(16.2)$ & $2(15.4)$ & $4(16.7)$ & 1.000 \\
\hline Sore muscles & $1(2.7)$ & $0(0.0)$ & $1(4.2)$ & 1.000 \\
\hline Diarrhea & $3(8.1)$ & $1(7.7)$ & $2(8.3)$ & 1.000 \\
\hline \multicolumn{5}{|l|}{ Clinical course, median (IQR), days } \\
\hline From illness onset to diagnosis & $4.5(2.3-8.8)$ & $6(4-13)$ & $4(4-7)$ & 0.098 \\
\hline From diagnosis to cured & $16(12-19)$ & $20(11.5-25.5)$ & $15(12-19)$ & 0.511 \\
\hline \multicolumn{5}{|l|}{ Duration of antiviral treatment } \\
\hline Median days (IQR) & $11(8-15)$ & $12(8-16)$ & $11(8-14)$ & 0.122 \\
\hline \multicolumn{5}{|l|}{ Duration of antibiotics treatment } \\
\hline Median days (IQR) & $13(10-17)$ & $15(12-20)$ & $10(3-14)$ & 0.001 \\
\hline \multicolumn{5}{|l|}{ Duration of hormone treatment } \\
\hline Median days (IQR) & $0(0-3)$ & $0(0-5)$ & $0(0-0)$ & - \\
\hline \multicolumn{5}{|l|}{ Complication } \\
\hline No & $32(86.5)$ & $11(84.6)$ & $21(87.5)$ & \\
\hline Respiratory failure & $4(10.8)$ & $2(15.4)$ & $2(8.3)$ & 0.742 \\
\hline Other type of pneumonia & $1(2.7)$ & $0(0.0)$ & $1(4.2)$ & 0.838 \\
\hline
\end{tabular}

In order to calculate the basic reproduction number $\left(R_{0}\right)$ among close contacts, we proposed the following SLI model [8]. The secondary attack rate was calculated as the number of secondary cases divided by the total number of household contacts. All analyses were conducted in the R software environment for statistical computing.

\section{Results}

3.1. Study Population. On 22 January, the first laboratoryconfirmed SARS-CoV-2 case in Suzhou was reported in a 37-year-old male who returned from Wuhan. By 22 February, 87 patients with SARS-CoV-2 infection were reported, 
TABLE 2: Results of laboratory tests of index and secondary cases in clusters.

\begin{tabular}{|c|c|c|c|c|}
\hline Characteristic & Total $(N=37, x \pm s)$ & Cluster index cases $(n=13, x \pm s)$ & Cluster secondary cases $(n=24, x \pm s)$ & $P$ value \\
\hline \multicolumn{5}{|l|}{ Infection-related biomarker } \\
\hline C-reaction protein $(\mathrm{mg} / \mathrm{L})$ & $13.37 \pm 16.04$ & $23.48 \pm 18.89$ & $7.91 \pm 11.28$ & 0.003 \\
\hline \multicolumn{5}{|l|}{ Blood biochemical test } \\
\hline Albumin $(\mathrm{g} / \mathrm{L})$ & $37.88 \pm 3.11$ & $35.85 \pm 2.81$ & $38.98 \pm 2.72$ & 0.002 \\
\hline Creatinine $(\mu \mathrm{mol} / \mathrm{L})$ & $58.00 \pm 21.26$ & $68.20 \pm 19.02$ & $52.48 \pm 20.69$ & 0.030 \\
\hline
\end{tabular}

including 50 (57.5\%) sporadic and 37 (42.5\%) clustered cases (Figure 1). The 37 clustered cases were generated from 13 clusters. The clusters included a mean of 4.8 contacts (range, 2 to 12). Of 13 clusters, 12 were household contacts, and the remaining one cluster was workmate contact. The median age of the 13 index patients was 49 years (range, 30 to 64 years). Of 37 patients, 20 (54.0\%) were male. Five (13.5\%) of the patients had comorbidities (Table S1).

3.2. Clinical Characteristics. On admission, 32 patients had clinical symptoms or signs, and the other 5 patients were asymptomatic, who had positive qRT-PCR results by testing all close contacts of confirmed cases. Of 32 patients with clinical symptoms, 23 (71.9\%) had fever or cough, and gastrointestinal symptoms (vomiting, nausea, and diarrhea) were prominent in five $(15.6 \%)$ patients. For blood counts, $36(97.3 \%)$ patients had normal or lower than average total white blood cell counts, and 24 (64.9\%) had normal or lower than average lymphocyte cell counts. By contrast, 16 (43.2\%) patients had substantially increased C-reactive protein levels. In addition, the increased procalcitonin levels were observed in $7(18.9 \%)$ patients. All patients were treated in isolation. The durations for antiviral treatment and antibiotics treatment were 2-28 days (median 5.5 days) and 3-21 days (median 12.5 days), respectively.

3.3. Index and Secondary Cases. The demographic and clinical characteristics of patients are indicators for viral virulence. In order to examine whether the virulence of SARSCoV-2 varied during human-to-human transmission, we compared the individual characteristics between index and secondary clustered cases. As shown in Table 1, the average age of index cases was higher than secondary cases (median age 49 years vs. $36 ; P=0.017$ ). The male ratio of index cases was significantly higher than that of secondary cases $(P=0.040)$. Additionally, the index cases were more likely to have fever than the secondary cases $(92.3 \%$ vs. $62.3 \% ; P$ $=0.015$ ). The interval between the onset of symptoms and final diagnosis for index cases (median 6 days) was longer than that for secondary cases (median 4 days), but the difference was not statistically significant $(P=0.098)$.

We further assessed the difference in blood routine and biochemical tests between index and secondary cases. As summarized in Table 2, the index cases were more likely to have increased CRP levels than secondary cases $(23.48 \mathrm{mg} / \mathrm{L}$ vs. $7.91 ; P=0.003)$. For biochemical test, the average creatinine level of index cases $(68.20 \mu \mathrm{mol} / \mathrm{L})$ was significantly higher than that of secondary cases
$(52.48 \mu \mathrm{mol} / \mathrm{L})$, whereas the index cases $(35.85 \mathrm{~g} / \mathrm{L})$ had lower level of albumin compared with the secondary cases $(38.98 \mathrm{~g} / \mathrm{L} ; P=0.002)$.

3.4. Epidemiological Characteristics of SARS-CoV-2 in Clusters. Figure 2 summarizes the epidemiological characteristics of SARS-CoV-2 clusters in our cohort. Overall, the $R_{0}$ values of clusters displayed a significantly declining trend over time for all clusters. The highest $R_{0}$ was observed in early stage of transmission in Cluster 10 , with a $R_{0}$ value of 8.05, followed by 6.60 in Cluster 1 and 5.55 in Cluster 3. At the end stage of transmission, each $R_{0}$ value ranged from 1.05 to 2.15 across clusters. Using full data on close contacts, the $\mathrm{RR}$ of infection in blood-related contacts of cases versus unrelated contacts was 1.60 for SARS-CoV-2 (95\% CI: 0.42-2.95).

\section{Discussion}

The outbreak of SARS-CoV-2 has triggered a rising global health emergency $[9,10]$. To our knowledge, this is the first report on its epidemiological characteristics among close contacts. Our results demonstrate that SARS-CoV-2 has great person-to-person transmission capability; however, the high $R_{0}$ values are only observed in early stage rather than the later stage of transmission. The substantial decline of $R_{0}$ values majorly attributes to limited contact of index and secondary cases in the community. Notably, the most important risk factor for acquiring SARS-CoV-2 infection in our clusters is close household contacts because of their prolonged exposure to the index cases in their house, which are relatively confined and poorly ventilated airspaces [11]. In addition, the adoption of strict precautions, such as closed community and delayed resumption of work [12], is essential to prevent its transmission in the workplace. Despite limited spread in household contacts, it would lead to exponential increase in burden of COVID-19. Therefore, we speculate that the isolation of the COVID-19 cases in socalled shelter or "Fang Cang" hospitals rather than the recommendation of cases with mild symptoms to be cared for at home by family members in Wuhan since early February has played an important role in the decline in new infections among household contacts, thereby accelerate control of SARS-CoV-2 epidemic.

Another interesting finding of this study is that the secondary COVID-19 cases are more prone to have mild symptoms than index cases. Symptomatic index cases are more likely to seek medical health care, thereby triggering active identification of secondary cases with mild symptoms. This 


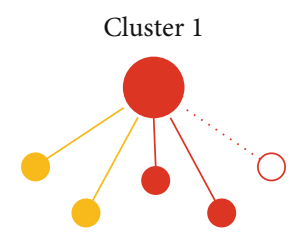

$\mathrm{R}_{0}: 6.60-2.15$; SAR: $80.0 \%$

Cluster 4

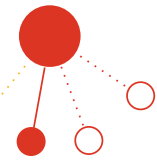

$\mathrm{R}_{0}: 2.55-1.28$; SAR: $25.0 \%$

Cluster 7

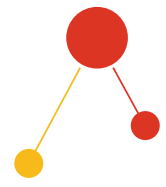

$\mathrm{R}_{0}$ : NA; SAR: $100.0 \%$

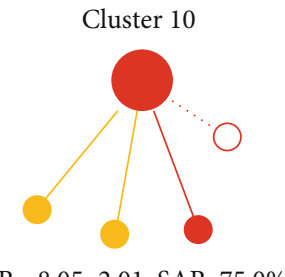

$\mathrm{R}_{0}$ : 8.05-2.01; SAR: $75.0 \%$
Cluster 2

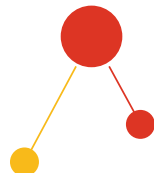

$\mathrm{R}_{0}$ : 1.22; SAR: $100.0 \%$

Cluster 5

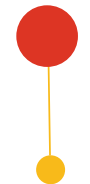

$\mathrm{R}_{0}$ : NA; SAR: $100.0 \%$

Cluster 8

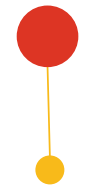

$\mathrm{R}_{0}$ : 1.39; SAR: $100.0 \%$

Cluster 11

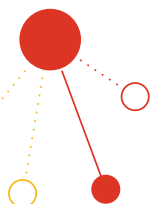

$\mathrm{R}_{0}: 2.55-1.28$; SAR: $25.0 \%$

Cluster 13
Cluster 3

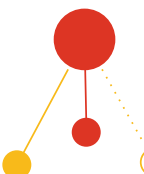

$\mathrm{R}_{0}$ : 5.55-1.85; SAR: $66.7 \%$

Cluster 6

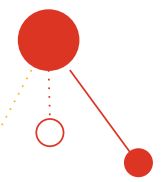

$\mathrm{R}_{0}: 1.39-1.15$; SAR: $33.3 \%$

Cluster 9

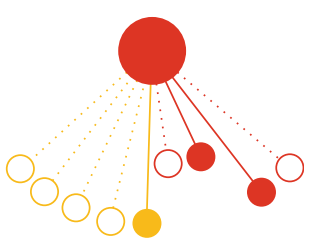

$\mathrm{R}_{0}: 1.78-1.05$; SAR: $33.3 \%$

Cluster 12

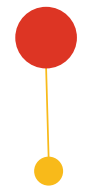

$\mathrm{R}_{0}$ : 1.39; SAR: $100.0 \%$

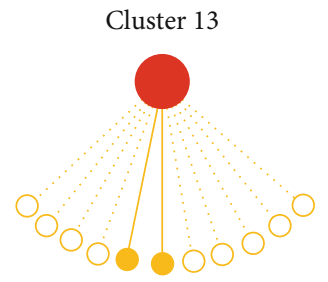

$\mathrm{R}_{0}: 1.73-1.15$; SAR: $18.7 \%$

Infected blood relative

Infected unrelated contact
Uninfected blood relative

Uninfected unrelated contact

FIGURE 2: Transmission of reported clusters of SARS-CoV-2 infections as differentiated by blood relatives and unrelated contacts. SAR: secondary attract rate. The red solid circle represents infected blood relatives. The red hollow circle represents uninfected blood relatives. The yellow solid circle represents infected unrelated contacts. The yellow hollow circle represents uninfected unrelated contacts. The distance between index and secondary case represents kin relationship.

phenomenon may be majorly associated with a higher likelihood of detecting asymptomatic secondary cases when actively contact tracing. In addition, previous studies have demonstrated that the loss in virulence that occurs following transmission may have implications for the capability of viruses to transmit disease $[13,14]$. Of note, as a new coronavirus of probable bat origin [15], SARS-CoV-2 crosses into new host population, and the continual introduction of new selective pressures would accumulate the accumulation of deleterious mutation conferring reduced virulence $[16,17]$. Further comparative genomic analysis of viruses isolated from index and secondary cases will bring new insights into molecular mechanism involving the loss of virulence during transmission.

We have shown that the ratio of male to female in secondary cases is markedly lower than that in index cases. 
Although this suggests a potential increased susceptibility to SARS-CoV-2 infections for female in clusters, the expression level of ACE2, the cell entry receptor of this novel coronavirus, shows no significant difference between gender groups $[18,19]$, which is opposed to our finding. After a careful analysis of the cases, a plausible explanation is that the high rate of male index case is associated with the gender-related change in secondary cases considering that the household contacts at the highest risk of subsequent infections are spouses due to their prolonged intimate contact. This hypothesis is supported by our finding that no increased $\mathrm{RR}$ of secondary infection in blood relatives is found compared with unrelated contacts. This epidemiological parameter is consistent with that of H7N9 but different from that of H5N1 [4]. More evidence is urgently needed to elucidate the varying host susceptibility across viruses.

Notably, approximate one-fifth of secondary cases in clusters are asymptomatic infections, which is higher than the proportion in SARS-CoV (13\%) [20]. The substantially high proportion of asymptomatic SARS-CoV-2 infections may be an indicator for reduced virulence than SARS-CoV, which corroborates previous findings that the mortality rate in patients with SARS-CoV-2 infection is lower than that previously seen in SARS patients $[21,22]$. In a recent case series, asymptomatic persons are potential sources of SARS-CoV-2 infections [23]. In this context, despite the lack of knowledge on the contribution of asymptomatic persons to transmission, the control measures will be hampered since they depend on the screening strategy of patients with clinical symptoms.

This study has several obvious limitations. First, despite enrolment of all clusters in Suzhou, the small sample size of our study necessitates cautious interpretation of our findings. Second, molecular assays currently available have low sensitivity during the initial stage of infection [24]. In view of the laboratory-confirmed cases included in this study, the exclusion of false-negative cases may lead to the underestimation of basic reproductive number. Finally, the genome sequence of each virus was not analyzed due to the biosafety risk concern. Hence, we could not identify nucleotide variations during transmission. Nevertheless, this first report on epidemiological characteristic of SARS-CoV-2 infections in clusters will help us to take effective actions to curb its transmission in close contacts.

In conclusion, our results demonstrate that SARS-CoV-2 has great person-to-person transmission capability; however, the high $R_{0}$ values are only observed in early stage rather than later stage of transmission. The secondary COVID-19 cases are more prone to have mild symptoms than index cases, which may be majorly associated with a higher likelihood of detecting asymptomatic secondary cases when actively contact tracing. There is no increased RR of secondary infection in blood relatives versus unrelated contacts. The high rate of asymptomatic SARS-CoV-2 infections highlights that the control measures will be hampered since they depend on the screening strategy of patients with clinical symptoms.

\section{Abbreviations}

SARS-CoV-2: Severe acute respiratory syndrome coronavirus 2
COVID-19: Coronavirus disease-19

R0: $\quad$ Basic reproduction number

SD: $\quad$ Standard deviations

RR: $\quad$ Relative risk.

\section{Data Availability}

The datasets generated and analyzed from the current study are not publicly available at this time as further analyses are ongoing but are available from the corresponding author on reasonable request.

\section{Conflicts of Interest}

The authors declare that they have no competing interests.

\section{Authors' Contributions}

Lin Yao, Peijun Tang, Hui Jiang, Binbin Gu, and Ping $\mathrm{Xu}$ contributed equally to this work.

\section{Acknowledgments}

This study was supported by the Social Development Project of Jiangsu Provincial Department of Science and Technology (BE2019673) and the High-level Health Talents in Jiangsu Province (LGY2019014). We thank all participating this study.

\section{Supplementary Materials}

Supplemental Table 1: demographic and clinical characteristics of laboratory-confirmed cases with SARS-CoV-2 infection in clusters. (Supplementary Materials)

\section{References}

[1] Q. Li, X. Guan, P. Wu et al., "Early transmission dynamics in Wuhan, China, of novel coronavirus-infected pneumonia," The New England Journal of Medicine, vol. 382, no. 13, pp. 1199-1207, 2020.

[2] J. F. Chan, S. Yuan, K. H. Kok et al., "A familial cluster of pneumonia associated with the 2019 novel coronavirus indicating person-to-person transmission: a study of a family cluster," Lancet, vol. 395, no. 10223, pp. 514-523, 2020.

[3] J. Liu, X. Liao, S. Qian et al., "Community transmission of severe acute respiratory syndrome coronavirus 2, Shenzhen, China, 2020," Emerging Infectious Diseases, vol. 26, no. 6, pp. 1320-1323, 2020.

[4] Y. Qin, P. W. Horby, T. K. Tsang et al., "Differences in the epidemiology of human cases of avian influenza A(H7N9) and $\mathrm{A}(\mathrm{H} 5 \mathrm{N1})$ viruses infection," Clinical Infectious Diseases, vol. 61, no. 4, pp. 563-571, 2015.

[5] S. Zhao, S. S. Musa, Q. Lin et al., "Estimating the unreported number of novel coronavirus (2019-nCoV) cases in China in the first half of January 2020: a data-driven modelling analysis of the early outbreak," Journal of Clinical Medicine, vol. 9, no. 2, p. 388, 2020.

[6] Y. Liu, A. A. Gayle, A. Wilder-Smith, and J. Rocklov, "The reproductive number of COVID-19 is higher compared to 
SARS coronavirus," Journal of Travel Medicine, vol. 27, no. 2, 2020

[7] P. Horby, H. Sudoyo, V. Viprakasit et al., "What is the evidence of a role for host genetics in susceptibility to influenza A/H5N1?," Epidemiology and Infection, vol. 138, no. 11, pp. 1550-1558, 2010.

[8] O. Diekmann, J. A. Heesterbeek, and J. A. Metz, "On the definition and the computation of the basic reproduction ratio $R_{0}$ in models for infectious diseases in heterogeneous populations," Journal of Mathematical Biology, vol. 28, no. 4, pp. 365-382, 1990.

[9] E. Alvarez, J. Donado-Campos, and F. Morilla, "New coronavirus outbreak. Lessons learned from the severe acute respiratory syndrome epidemic," Epidemiology and Infection, vol. 143, no. 13, pp. 2882-2893, 2015.

[10] WHO, "Global surveillance for human infection with coronavirus disease (COVID-19)," 2020, https://www.who.int/ emergencies/diseases/novel-coronavirus-2019/situationreports.

[11] J. J. Nania, J. Skinner, K. Wilkerson et al., "Exposure to pulmonary tuberculosis in a neonatal intensive care unit: unique aspects of contact investigation and management of hospitalized neonates," Infection Control and Hospital Epidemiology, vol. 28, no. 6, pp. 661-665, 2007.

[12] S. Chen, J. Yang, W. Yang, C. Wang, and T. Barnighausen, "COVID-19 control in China during mass population movements at New Year," Lancet, vol. 395, no. 10226, pp. 764$766,2020$.

[13] N. Dharmayanti, S. W. Thor, N. Zanders et al., "Attenuation of highly pathogenic avian influenza A(H5N1) viruses in Indonesia following the reassortment and acquisition of genes from low pathogenicity avian influenza A virus progenitors," Emerging microbes \& infections, vol. 7, no. 1, p. 147, 2018.

[14] S. Mueller, D. Papamichail, J. R. Coleman, S. Skiena, and E. Wimmer, "Reduction of the rate of poliovirus protein synthesis through large-scale codon deoptimization causes attenuation of viral virulence by lowering specific infectivity," Journal of Virology, vol. 80, no. 19, pp. 9687-9696, 2006.

[15] P. Zhou, X. L. Yang, X. G. Wang et al., "Addendum: A pneumonia outbreak associated with a new coronavirus of probable bat origin," Nature, vol. 588, no. 7836, p. E6, 2020.

[16] R. A. Weiss, "Virulence and pathogenesis," Trends in Microbiology, vol. 10, no. 7, pp. 314-317, 2002.

[17] S. F. Elena, R. Sanjuan, A. V. Borderia, and P. E. Turner, "Transmission bottlenecks and the evolution of fitness in rapidly evolving RNA viruses," Infection, Genetics and Evolution, vol. 1, no. 1, pp. 41-48, 2001.

[18] Y. M. Qin, C. Y. Hu, Y. Pang, A. J. Kastaniotis, J. K. Hiltunen, and Y.X. Zhu, "Saturated very-long-chain fatty acids promote cotton fiber and Arabidopsis cell elongation by activating ethylene biosynthesis," The Plant Cell, vol. 19, no. 11, pp. 36923704, 2007.

[19] X. Xudong, C. Junzhu, W. Xingxiang, Z. Furong, and L. Yanrong, "Age- and gender-related difference of ACE2 expression in rat lung," Life Sciences, vol. 78, no. 19, pp. 2166-2171, 2006.

[20] A. Wilder-Smith, M. D. Teleman, B. H. Heng, A. Earnest, A. E. Ling, and Y. S. Leo, "Asymptomatic SARS coronavirus infection among healthcare Workers, Singapore," Emerging infectious diseases, vol. 11, no. 7, pp. 1142-1145, 2005.
[21] J. W. Chan, C. K. Ng, Y. H. Chan et al., "Short term outcome and risk factors for adverse clinical outcomes in adults with severe acute respiratory syndrome (SARS)," Thorax, vol. 58, no. 8, pp. 686-689, 2003.

[22] W. J. Guan, Z. Y. Ni, Y. Hu et al., "Clinical characteristics of coronavirus disease 2019 in China," The New England Journal of Medicine, vol. 382, no. 18, pp. 1708-1720, 2020.

[23] X. Pan, D. Chen, Y. Xia et al., "Asymptomatic cases in a family cluster with SARS-CoV-2 infection," The Lancet Infectious Diseases, vol. 20, no. 4, pp. 410-411, 2020.

[24] J. S. Ellis and M. C. Zambon, "Molecular diagnosis of influenza," Reviews in Medical Virology, vol. 12, no. 6, pp. 375389, 2002. 